\title{
Second Metatarsophalangeal Joint Pigmented Villonodular Synovitis: A case report
}

\author{
by John A. Rialson, DPM ${ }^{1}$, Eric J. Heit, DPM, FACFAS ${ }^{2}$
}

The Foot and Ankle Online Journal 4 (9): 2

Pigmented Villonodular Synovitis (PVNS) is an uncommon condition of the foot. PVS of the foot accounts for $2 \%$ of all cases. We present the case of a 26 year-old male who was found to have pigmented villonodular synovitis of the second metatarsophalangeal joint. Although a high incidence is reported in the literature, the patient underwent surgical excision with no known reoccurrence to date. Findings from magnetic resonance imaging, surgical presentation and literature review is presented.

Key words: Pigmented villonodular synovitis, metatarsophalangeal joint, foot surgery, soft tissue mass.

Accepted: August, 2011

Published: September, 2011

This is an Open Access article distributed under the terms of the Creative Commons Attribution License. It permits unrestricted use, distribution, and reproduction in any medium, provided the original work is properly cited. @The Foot and Ankle Online Journal (www.faoj.org), 2011 All rights reserved.

$\mathrm{P}$ ain to the second metatarsophalangeal joint (MTP) is a common complaint encountered in the foot and ankle surgeon's office. While reasons for this presentation are vast, a soft tissue mass could be a cause of this pain. Proper work up of the patient that includes a thorough history and physical, basic radiographs, and advanced imaging studies can aid in an accurate diagnosis.

Pigmented villonodular synovitis (PVNS) is a fairly rare disease. The incidence of PVNS is 1.8 patients per million people. ${ }^{1}$ Pigmented villonodular synovitis is a proliferative disorder of synovial tissue in the joint, tendon sheath, and/or bursa.

Address correspondence to: Swedish Podiatric Surgical Residency Program, Swedish Medical Center, 747 Broadway Seattle, WA 98122

\footnotetext{
${ }^{1}$ Submitted while Chief Resident, Swedish Podiatric Surgical Residency Program, Swedish Medical Center, Seattle, WA

${ }^{2}$ Attending Surgeon, Swedish Podiatric Surgical Residency Program, Virginia Mason Medical Center, Seattle, WA
}

The common locations for PVNS are the knee, hip and ankle. Two percent of these lesions are in the foot. ${ }^{2}$ Bone and joint destruction can occur with this disease which makes early, accurate diagnosis and treatment essential. Furthermore, despite appropriate management by surgical excision ${ }^{3}$, PVNS has a high recurrence rate. In the foot and ankle, recurrence rate has been reported as $14.3 \%{ }^{4}$

\section{Case Report}

A 26 year-old male presented to the institution of the senior author with right foot pain that he had for a few weeks. The patient was unsure of the cause for the pain, but did remember feeling a pop to his right foot while performing yoga. He initially did not seek care as he was not in a lot of discomfort, but progressively his second MTP joint became more painful. 


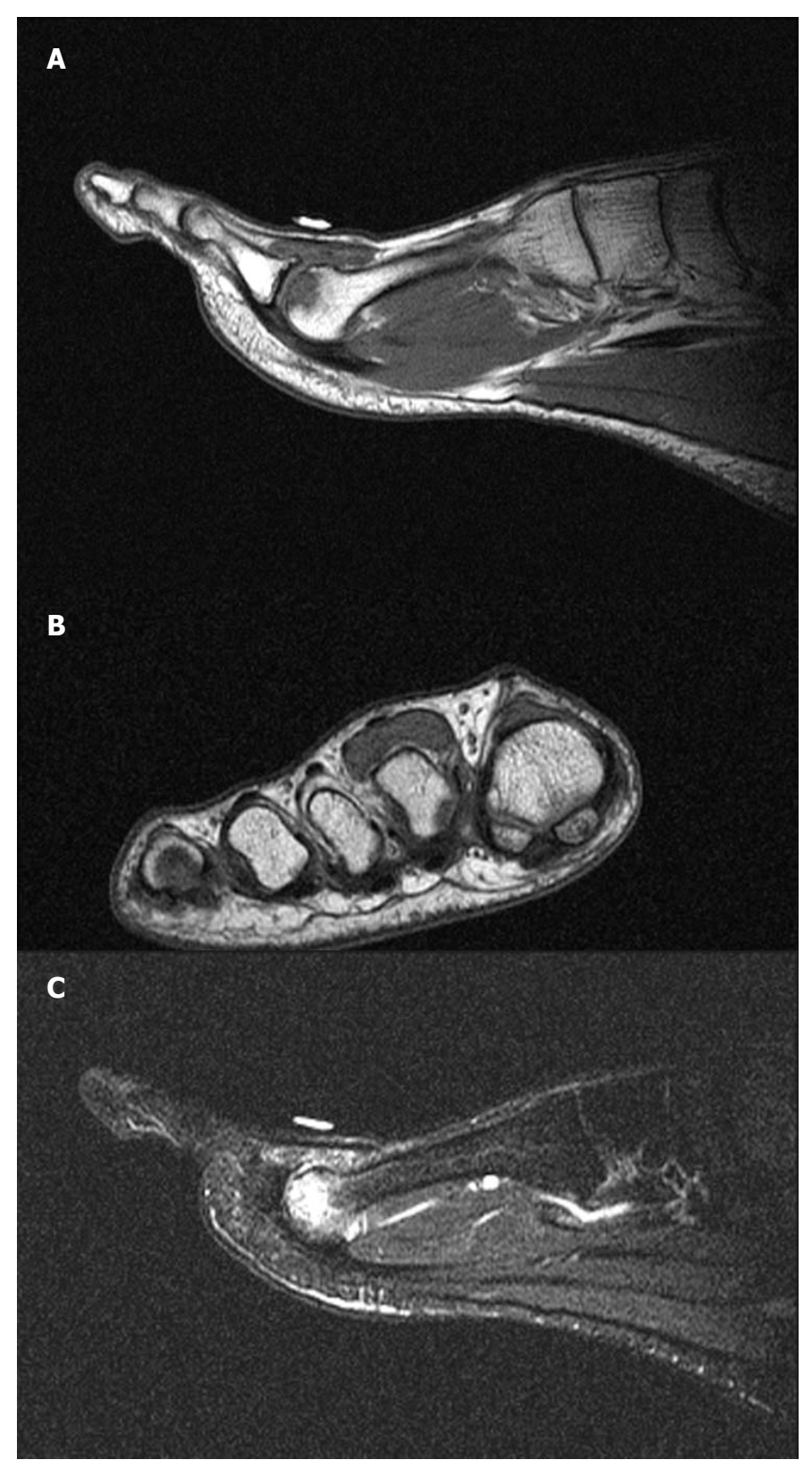

Figures $1 \mathrm{ABC}$ MR T1 image of the second MTP joint showing the soft tissue mass. (A) An Axial image showing the mass to the second metatarsal. (B) A STIR image of the soft tissue mass as well as showing the signal to the metatarsal head. (C)

The patient was able to point to his second MTP joint as is primary location of pain. Upon presentation, the area was so painful that he was using a crutch to take the pressure off the area. No other forms of treatment have been attempted prior to evaluation.
Clinical evaluation of the area revealed mild edema over the dorsal aspect of the second MTP joint without erythema or warmth. There was no significant deformity or instability, and neurovascular status was intact with an otherwise benign exam. Initial diagnostic imaging consisted of anteroposterior, medial oblique, and lateral foot radiographs, which were normal. There were no obvious signs of Freiberg's infraction. The only finding was a very mild joint abnormality with a subchondral cyst that was appreciated on the oblique view.

The initial diagnosis was capsulitis of the second MTP joint with possible osteochondral defect. Initial treatment consisted of CAM boot application and discussion of further imaging if not improving. The plan was to attempt this treatment for three weeks and reevaluate his foot with the option to obtain an magnetic resonance imaging (MRI).

The patient presented for follow up after the initial visit and stated that overall his condition felt worse. After the initial treatment, he experienced about five days of pain relief but developed increased pain since the initial relief. Once again, pain was localized to the second MTP joint. Clinically, there was a reduction of swelling to the area; however, with the decrease in swelling, a subcutaneous palpable mass was now present on the dorsal aspect of the second MTP joint. The contour of the metatarsal could also then be palpated with no obvious findings. The decision was then to obtain an MRI scan of the right foot.

The official findings of the MRI are as follows: “... markedly abnormal thickening and enhancement of the synovium surrounding the second metatarsophalangeal joint, with minimal associated joint fluid seen. Within the second MTP joint, there is abnormal signal within the metatarsal head with potential collapse of the cortical surface." (Figs. 1A, 1B and 1C) The radiologist's impression was a differential consideration to include a severe synovitis or other primary synovial abnormality such as PVNS. Also mentioned was no involvement of other joints to suggest a polyarticular arthropathy such as rheumatoid arthritis. 


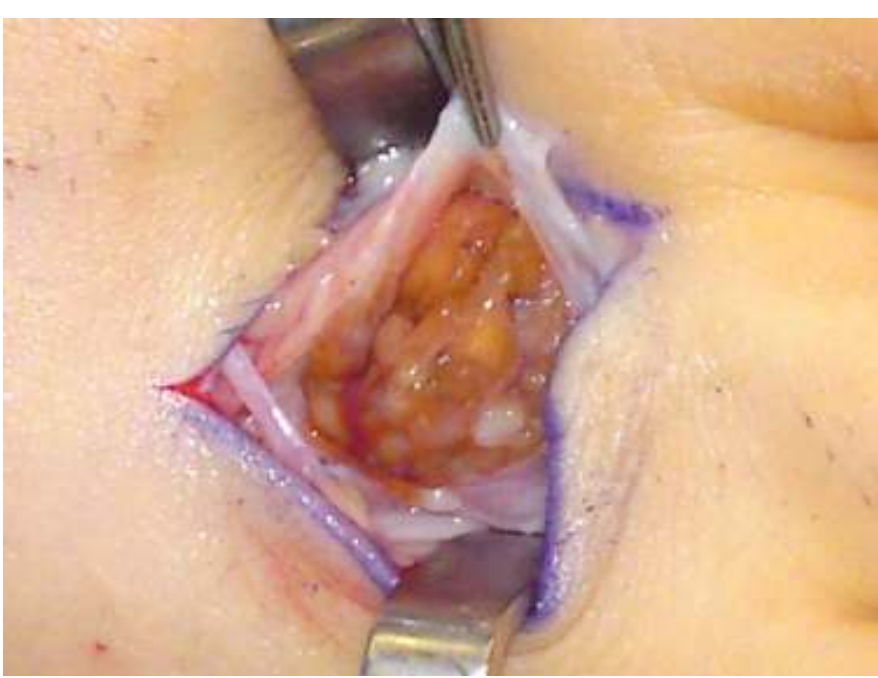

Figure 2 An intraoperative picture of the soft tissue mass consistent with PVNS.

With the above findings, the results of the MRI and treatment options were discussed with the patient. A surgical excision was planned and performed without complications. Intraoperatively after the incision and initial dissection, a lobular pigmented soft tissue mass, consistent with PVNS, was encountered. (Fig. 2) The soft tissue mass was dissected, removed, and sent for pathologic examination. (Fig. 3) Upon removing the mass, a cartilage defect to the second metatarsal head was appreciated. (Fig. 4) This finding was anticipated with the MRI but with carefully examination was a substantial deficit. The decision was made to fenestrate the head of the second metatarsal to promote fibrocartilage growth. (Fig. 5)

The pathology report confirmed the diagnosis consistent with PVNS. He had an uneventful post operative course and at last exam had no tenderness with palpation. The second digit was in a plantigrade position and had good range of motion across the MTP joint. The patient is now over one year after the procedure and there has been no known reoccurrence to date.

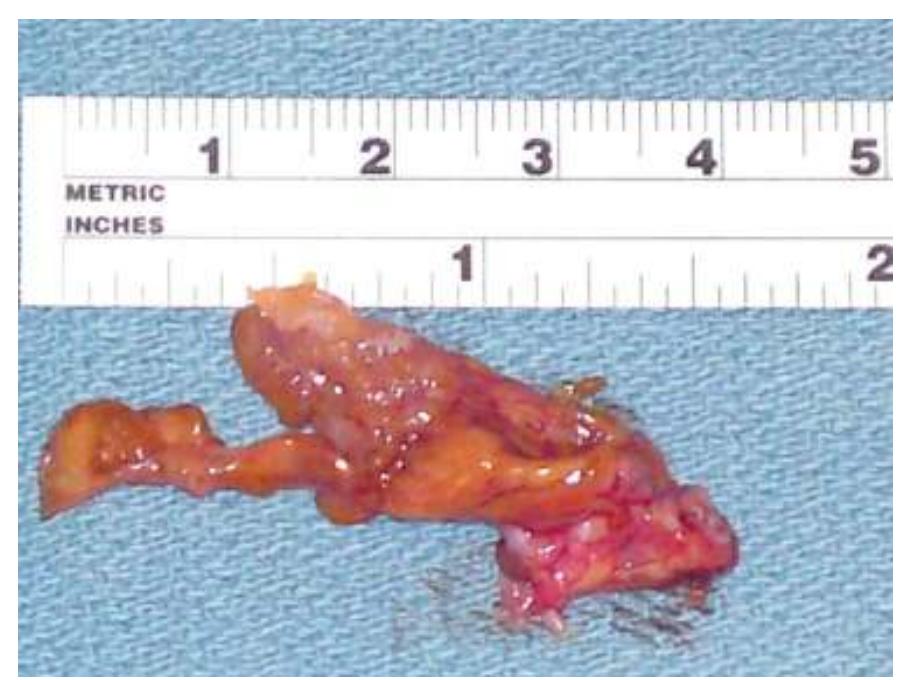

Figure 3 The soft tissue mass removed from the second MTP joint and sent for examination.

\section{Discussion}

Soft tissue masses of the foot and ankle often present with complaints of pain and/or irritation with footwear. Pigmented villonodular synovitis, while most common in the knee, hip and ankle, can present in the foot, although they are most commonly seen in the rearfoot and ankle ${ }^{2}$. Nabeshima, et al., does describe a case report of PVNS to the first MTP joint. ${ }^{9}$

Carpintero, et al., ${ }^{2}$ reported eight cases of PVNS in the foot. Five of the eight cases were reported in the rearfoot and the remaining three cases in the forefoot. The locations they reported were the talus, first cuneiform, first and fifth metatarsals, and middle phalanx. Interestingly, they also reported that there was radiographic bone involvement in six patients.

Similarly, Ghert, et al., ${ }^{5}$ documented the rarity of PVNS in the foot and ankle with a review of six cases from 1978-1997. Of the six of their cases, two were isolated to the ankle joint. The remaining four cases involved the subtalar joint, midfoot and two cases in the metatarsal region. Lisfranc amputation was a treatment for one of the cases. 


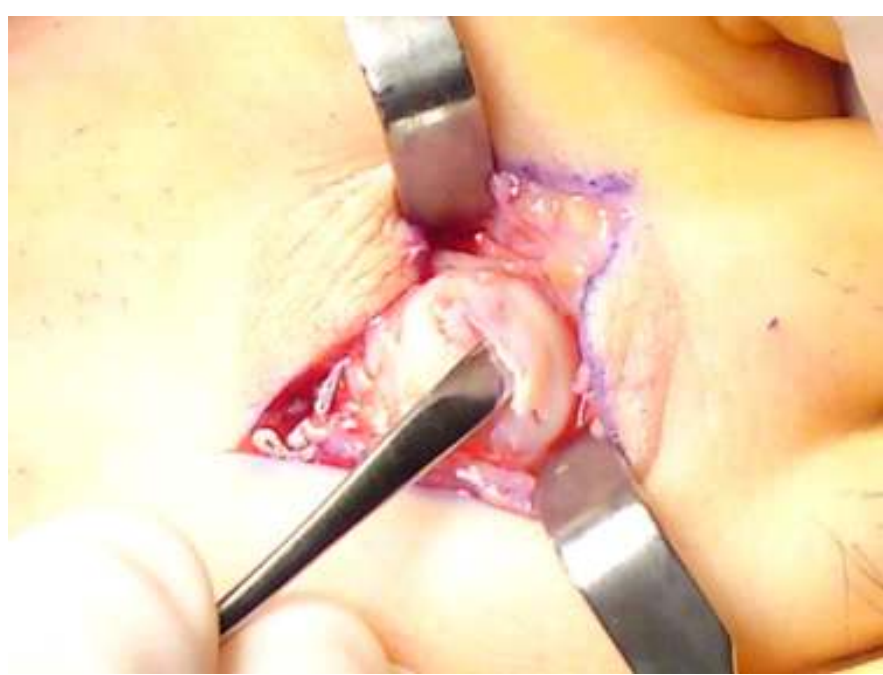

Figure 4 Operative finding of the cartilage defect to the head of the second metatarsal.

The invasive nature of PVNS is also an important aspect. This is described with bone involvement being found in seven of eight cases that Rochwerger et al. reported between 1981 and 1997. Surgical treatment consisted of arthrodesis in six cases, one ankle synovectomy, and one toe amputation.

In our patient's case, as well as in the literature, the use of MRI aids in the diagnosis of PVNS. The description of PVNS on MRI may have certain findings consistent with other synovial lesions. On T1-weighted images, the lesions will have low or intermediate signal intensity. Pigmented villonodular synovitis will also demonstrate low signal intensity with T2-weighted and gradient-echo images. The theory is that the hemosiderin in PVNS produces this low signal intensity on T2-weighted imaging. ${ }^{7}$

The final diagnosis of PVNS can be confirmed with pathological examination. Specimens may appear villous, nodular, or villonodular with often prominent hemosiderin deposition seen in most cases. ${ }^{8}$ Histological findings of PVNS are hyperplastic synovium, giant cells, and mononuclear histiocytes with varying extent of hemosiderin deposition., ${ }^{3,7}$

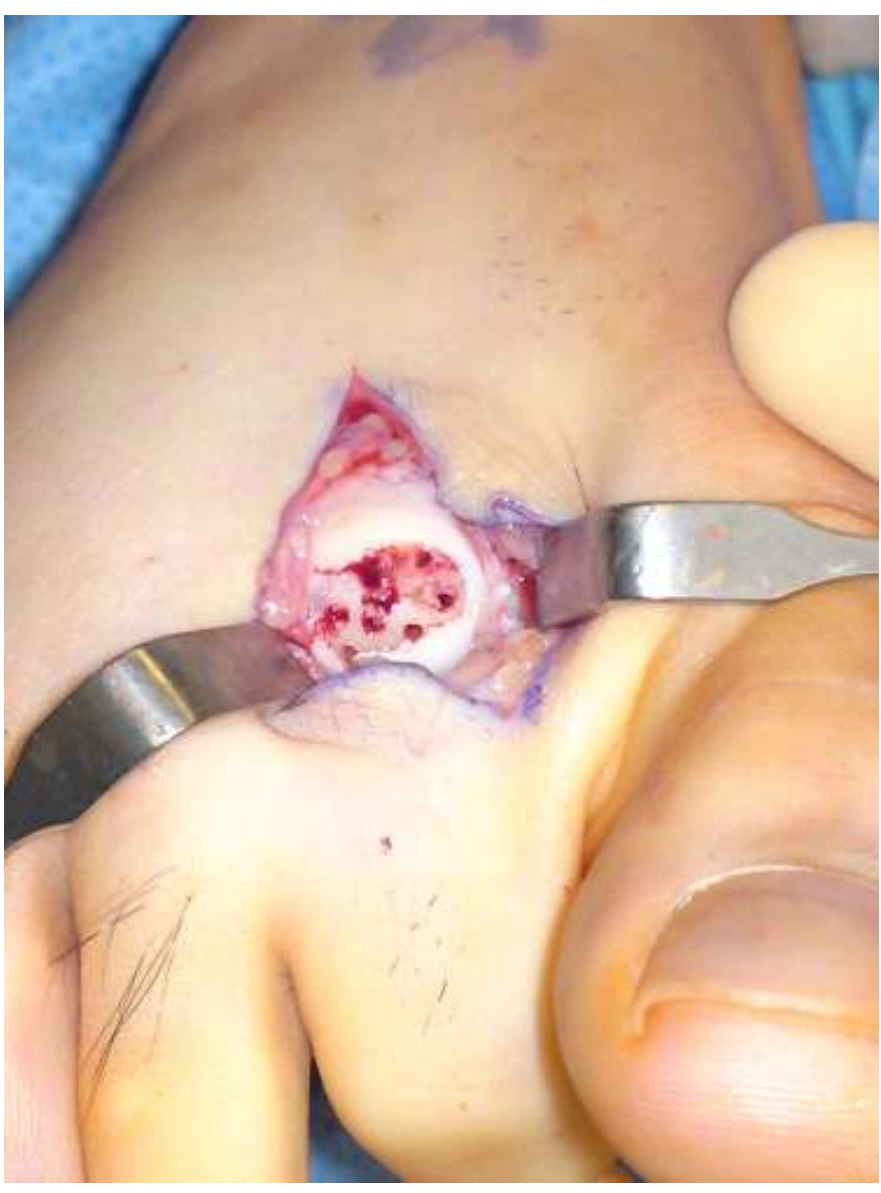

Figure 5 Operative photo showing the cartilage defect to the second metatarsal after fenestration.

While the literature states the PVNS is rare in the foot and of unknown etiology ${ }^{2}$, some theories of etiology that are often debated are an inflammatory process, neoplasia, or lipid metabolism disorder. The neoplastic theory is becoming more strongly supported by the discovery of cytogenetic aberrations. Mild repetitive trauma with hemorrhage into the joint also could explain the inflammatory nature and the hemosiderin of PVNS. One could speculate that this may be the origin in our patient with his yoga history. 


\section{References}

1. Myers BW, Masi AT. Pigmented villonodular synovitis and tenosynovitis: a clinical epidemiologic study of 166 cases and literature review. Medicine 1980 59: 223-238.

2. Carpintero P, Gascon E, Mesa M, Mesa M, Jimenez C, Lopez

U. Clinical and radiographic features of pigmented villonodular synovitis of the foot: Report of eight cases. JAPMA 2007 97: 415-419.

3. Brien EW, Sacoman DM, Mirra JM. Pigmented villonodular synovitis of the foot and ankle. Foot Ankle Int 2004 25: 908 913.

4. Sharma H, Jane MJ, Reid R. Pigmented villonodular synovitis of the foot and ankle: Forty years of experience from the Scottish Bone Tumor Registry. J Foot Ankle Surgery 2006 45: 329-336.

5. Gher MA, Scully SP, Harrelson JM. Pigmented villonodular synovitis of the foot and ankle: A review of six cases. Foot Ankle Int 1999 20: 326-330.

6. Rochwerger A, Groulier P, Curvale G, Launay F. Pigmented villonodular synovitis of the foot and ankle: A report of eight cases. Foot Ankle Int 1999 20: 587-590.

7. Llauger J, Palmer J, Monill JM, Franquet T, Bague S, Roson Nl. MR imaging of benign soft-tissue masses of the foot and ankle. Radiographics 1998 18: 1481-1498.

8. Murphy MD, Rhee JH, Lewis RB, Fanburg-Smith JC, Flemming DJ, Walker EA. Pigmented villonodular synovitis: Radiologic-pathologic correlation. Radiographics 2008 28:14931518.

9. Nabeshima Y, Mori H, Mitani M, Nagura I, Ozaki A, Fujii H, Doita M. Diffuse pigmented villonodular synovitis in the metatarsophalangeal joint of the Hallux: A Case Report. J Foot Ankle Surgery 2009 48: 573-576. 